\title{
Journal of Aging and Physical Activity Celebrates Its 30th Year With a Virtual Special Issue: Reflections on the First $\mathbf{3 0}$ Years From the Editors
}

\author{
Wojtek Chodzko-Zajko, ${ }^{1}$ Debra J. Rose, ${ }^{2}$ Diane E. Whaley, ${ }^{3}$ Philip D. Chilibeck, ${ }^{4}$ and Samuel R. Nyman ${ }^{5}$ \\ ${ }^{1}$ Graduate College, University of Illinois at Urbana-Champaign, Champaign, IL, USA; ${ }^{2}$ Department of Kinesiology and Center \\ for Successful Aging, California State University, Fullerton, Fullerton, CA, USA; ${ }^{3}$ Department of Leadership, Foundations, and Policy, \\ University of Virginia, Charlottesville, VA, USA; ${ }^{4}$ College of Kinesiology, University of Saskatchewan, Saskatoon, SK, Canada; \\ ${ }^{5}$ Bournemouth University Clinical Research Unit, Department of Medical Science and Public Health, Bournemouth University, \\ Dorset, United Kingdom
}

\begin{abstract}
This is the first of a series of virtual special issues. Each special issue will feature previously published articles in Journal of Aging and Physical Activity (JAPA). These online-only collections will serve to highlight the contribution of the journal to advancing science, policy, and practice. We hope they inspire researchers to publish their innovative and impactful work in $J A P A$.

When the first issue of JAPA was published in January 1993, the Editorial Board's primary goal was to establish the first interdisciplinary journal that would publish original research reports, scholarly reviews, and professional application articles that pertain to the relationship between physical activity and the aging process. We hoped to stimulate the submission of articles that could contribute to an understanding of (a) the impact of physical activity on physiological, psychological, and social aspects of older adults, and (b) the effect of advancing age and/or the aging process on physical activity patterns and attitudes toward physical activity in older adults. In this virtual special issue, the serving editors of $J A P A$ have selected 19 articles for inclusion. These articles, and their reasons for inclusion, are summarized below.
\end{abstract}

There can be little doubt that the journal was successful in achieving the above goal during its founding decade. Among the notable highlights of the first 10 years were the publication of keynote addresses from two world congresses, Heidelberg, 1996, and Orlando, 1999, and four U.S. national conferences on Aging and Physical Activity. In addition, in 1997, we published the World Health Organization's Heidelberg Guidelines for Promoting Physical Activity Among Older Persons. In 2001, JAPA issued a special supplement containing The National Blueprint: Increasing Physical Activity Among Adults Age 50 and Older, a major national planning document developed to serve as a guide for multiple organizations, associations, and agencies, to support their planning work related to increasing physical activity among America's aging population.

We believe the five articles selected from 1993 to 2002 reflect the breadth and depth of the scholarship published in JAPA. ${ }^{11}$ Waneen Spirduso (1994) is without question one of the pioneers of research on physical activity and aging, and her retrospection and vision for the future rings as true today as it did when it was first published almost 30 years ago. Edward McAuley and David Rudolph (1995) provide an excellent example of how rigidly controlled randomized clinical trials can increase our understanding of the benefits of physical activity. Similarly, but quite differently, Sandra O'Brien Cousins and Patricia Vertinsky (1995) remind us of the insight and wisdom to be gained by inviting older persons to tell us their stories. In 1997, we published the World Health
Organization Heidelberg Guidelines for Promoting Physical Activity among Older Persons (1997). These guidelines precipitated an explosion of interest in the promotion of physical activity for older persons around the globe. Finally, Roberta E. Rikli and C. Jessie Jones (1999) published their groundbreaking work that led to the development of the Senior Fitness Test, perhaps the most frequently used field test of functional fitness in use today.

We have selected four articles from 2003 to 2006. Sheppard et al. (2003) summarized the outcomes of a consensus conference attended by representatives from $>50$ organizations tasked with identifying strategic priorities for advancing the National Blueprint: Increasing Physical Activity Among Adults Age 50 and Older. Not surprisingly, Wojtek Chozdko-Zajko (founding editor of $J A P A$ ), assumed a major leadership role in advancing the first ever nationwide effort to "identify some of the societal barriers to physical activity participation in midlife and older adults and to outline specific strategies for overcoming them" (p. 287). The selected articles for 2004 and 2005 were both awarded Publication of the Year honors by a committee comprised of three editorial board members. The Estabrooks et al.'s (2004) article was lauded for its methodical application of an existing theory-driven approach to group dynamics to understanding and identifying the characteristics of motivational leaders of physical activity programs for older adults. The 2005 winning publication, also awarded to a research group led by Estabrooks, was considered "an exemplar of how to design and conduct a pragmatic exploratory trial and of how to publish a series of studies that tell an interesting and important research story."22 An article published by Stewart et al. in 2006 was selected for its multilevel, community-based approach to identifying meaningful ways to diffuse a research-based physical activity program The Community Healthy Activities Model Program for Seniors (CHAMPS) in underserved communities. The study's findings can guide communities in their efforts to reduce socioeconomic and racial/ethnic health disparities among older adults through increased physical activity.

The papers we selected from 2012 to 2016 are representative of key areas of emphasis for the serving editor: What constitutes physical activity, how stereotypes of aging might impact physical activity, the importance of taking a developmental approach in studying aging, and how to make our research more approachable to practitioners. Chen and Janke (2012) found a significant relationship between gardening (including more vigorous behaviors such as digging) and balance/gait. This signaled a turn in JAPA to include a more comprehensive view of physical activity. Chalebaev 
et al. (2013) built on the work of aging researchers like Levy, who have found that negative stereotypes often embraced by older adults (e.g., aging as decline) impact their behaviors. In this study, the authors relate aging stereotypes to physical activity behavior, developing a measure to assess the role of stereotypes in decisions to engage in physical activity. The third paper presents an example of a developmental approach to understanding physical activity in older adults. Stone and Baker (2014) found differences across the older adult age range in what should be the focus of physical activity interventions; that is, individuals in their 60 s are likely to have different interests, abilities, and motives for their exercise behavior than those in their 80s. The paper by Adamo, Talley, and Goldberg (2015) is also explicitly developmental. They showed both differences across age groups (e.g., in their ability to perform functional fitness tasks) and similarities (e.g., declines in lower body strength) across the $40+$ years of older adulthood. In addition, this paper provided clear, specific recommendations for health care professionals regarding the value of normative versus norm-referenced scores. The article by Middleton et al. (2016) continues the theme of providing useful research findings and practical implications for health care providers. They compared the utility of using self-selected walking speed, a simple, no-cost measure, with accelerometer data as a way of predicting daily ambulatory activity. The authors concluded that self-selected walking speed can be used as a screening tool to identify those who may need to increase their activity rates, thus providing practitioners with a solid alternative to wearable devices.

We have selected five papers from 2016 to 2019. The effect of the built environment on physical activity was an important research theme during this period. Moran et al. (2017) used a mixed-methods approach to determine barriers and facilitators to neighborhood walking. Barriers included pedestrian infrastructure (cracked/broken sidewalks, obstacles blocking sidewalkse.g., parked cars, overgrown hedges, lack of curb cuts and crosswalks), esthetics (neglected buildings), environmental quality (litter, heavy traffic), and crime. Facilitators included food-related and shopping destinations, benches, and esthetics (e.g., beautiful architecture). The recognition that sedentary behavior is important for health of older adults also emerged as an important theme. Several large epidemiological studies showed strong relationships between sedentary behavior, health, and mortality. Older adults sitting $>4 \mathrm{hr} /$ day had $76 \%-82 \%$ higher prevalence of the presence of $\geq 2$ chronic diseases (Riberio et al., 2018). Over 6 years, older adults with high television viewing time had increased mortality (hazard ratio $=1.65$ ) with the relationship especially strong for those with hypertension and diabetes (hazard ratio $=3.54$ ) (Lemes et al., 2019). Therefore, it is important that interventions be developed to promote breaking up of sitting time in older adults. Insight into the benefit of physical activity on aging was gained by examining the very old. Pes et al. (2018) found a surprisingly high physical activity level from accelerometer tracking of nonagenarians from a Sardinian village famous for longevity. Furthermore, over a 6-year longitudinal study, physical activity level was strongly associated with decreased mortality in 80- to 90-year-old participants (Mace Firebaugh et al., 2018). Physical activity therefore benefits even the oldest of the old.

In conclusion, we hope that this selection of papers highlights the breadth and depth of scientific understanding that has been advanced in JAPA. The articles exemplify quality work across disciplines, populations, and different levels (working at the individual level and at the policy level), to advance theory, methods, and practice. We thank all our authors and reviewers who have contributed to the journal over the years. It is this collaboration among many colleagues across the globe that has made JAPA a success over the past 30 years. We look forward to an increasingly bright future for JAPA's contribution to science, policy, and practice.

Wojtek Chodzko-Zajko (Founding Editor: 1993-2002)

Debra J. Rose (2003-2006)

Diane E. Whaley (2012-2016)

Philip D. Chilibeck (2016-2019)

Samuel R. Nyman (2020-present)

\section{Notes}

1. JAPA papers cited in-text are included in the virtual special issue.

2. Original research article award winner and reviewer of the year for 2005. (2006). Journal of Aging and Physical Activity, 14(1), 1-2. Print. 